PROCEEDINGS OF THE

AMERICAN MATHEMATICAL SOCIETY

Volume 135, Number 12, December 2007, Pages 4087-4098

S 0002-9939(07)08972-1

Article electronically published on September 12, 2007

\title{
ON THE TOPOLOGY OF THE BOUNDARY OF A BASIN OF ATTRACTION
}

\author{
J. J. SÁNCHEZ-GABITES AND J. M. R. SANJURJO
}

(Communicated by Alexander N. Dranishnikov)

\begin{abstract}
Suppose $\varphi: M \times \mathbb{R} \longrightarrow M$ is a continuous flow on a locally compact metrizable space $M$ and $K$ is an (asymptotically stable) attractor. Let $D=\partial \mathcal{A}(K)$ be the boundary of the basin of attraction of $K$. In the present paper it will be shown how the Conley index of $D$ plays an important role in determining the topological nature of $D$ and allows one to obtain information about the global dynamics of $\varphi$ in $M$.
\end{abstract}

\section{INTRODUCTION}

1.1. General setting. Our general setting will be the following: consider a continuous flow $\varphi$ on a locally compact phase space $M$ which is an ANR. Let $K$ be an attractor, whose basin of attraction we shall denote $\mathcal{A}(K)$ : then it is known that the inclusion $i: K \hookrightarrow \mathcal{A}(K)$ is a shape equivalence and $K$ has the shape of a finite polyhedron (see [3, [10, 12] or [19]). The clue why results such as this can be obtained is, essentially, that the flow provides a way of collapsing $\mathcal{A}(K)$ onto $K$ (this line of reasoning is recorded here in Lemma 2.5). More generally, moving points along their trajectories is a standard technique in the realm of continuous dynamical systems, albeit it has natural dynamical limitations. For example, if we denote $D=\partial \mathcal{A}(K)$ the boundary of the region of attraction, it is clear that $D$ constitutes a dynamical barrier which isolates $\mathcal{A}(K)$ from $M-\overline{\mathcal{A}(K)}$ and the flow cannot be used any longer to obtain information relating those sets.

However, it is clear that a thorough study of $D$ is a first step towards results enabling the passage from local information near the attractor $K$ to a global description of the flow. This is especially well exemplified by Proposition 3.3. where a Conley index condition on $D$ renders a complete picture of the dynamics in compact phase spaces. Besides, it turns out that in spite of the known complicated dynamics which can take place in $D$, the same condition is enough to guarantee a nice topological structure on $D$, namely having polyhedral shape (Theorem 2.6). The relevance of a careful analysis of the boundary of a basin of attraction has been also pointed out in other papers (see, for instance [17]). Let us remark that the study of sections of flows is a classical subject in the field of dynamical systems and the boundary of the basin of an attractor $K$ can be thought of (at least in certain cases) as a limit of sections of $\mathcal{A}(K)-K$ which in backwards time approximate

Received by the editors March 22, 2006 and, in revised form, September 15, 2006.

2000 Mathematics Subject Classification. Primary 54H20, 55P55, 58F12.

The authors were partially supported by DGI.

(C)2007 American Mathematical Society Reverts to public domain 28 years from publication 
$D$. Sections enjoy good topological properties, so it is natural to ask when the boundary of $\mathcal{A}(K)$ also does.

Since the topology of $D$ is invariant under conjugation, results like the ones presented here could be helpful in matters related to classification of flows, especially bearing in mind that the polyhedral shape of $D$ implies that its Cech homology and cohomology groups are finitely generated. This makes more operational the computational tools provided by algebraic topology.

In order to place our discussion in an appropriate context, some examples are included to make it evident that in a general case the boundary of $\mathcal{A}(K)$ can be strongly misbehaved from a topological point of view.

Finally, we would like to thank the referee for his helpful remarks and Rafael Ortega for stimulating conversations.

1.2. Preliminary definitions and results. We shall briefly recall here the relevant definitions and, at the same time, some basic results to be used. Please note that, due to the lack of a universally accepted notation, ours can depart from the one used in the cited references. In particular, let us adopt from the very beginning the shorthand $x \cdot t$ for $\varphi(x, t)$.

For any subset $S \subseteq M$, its alpha- and omega-limit sets are defined by $\alpha(S)=$ $\bigcap_{t \leq 0} \overline{S \cdot(-\infty, t]}$ and $\omega(S)=\bigcap_{t \geq 0} \overline{S \cdot[t,+\infty)}$ respectively; they are always closed and invariant. An important (but easy to prove) fact concerning them is that, if $S$ is a continuum and a limit set is compact, then it is connected.

A compact invariant set $K$ is an (aymptotically stable) attractor if it posseses a neighbourhood $U$ such that $K=\omega(U)$, and in this case its basin of attraction $\mathcal{A}(K)=\{x \in M: \emptyset \neq \omega(x) \subseteq K\}$ is an open neighbourhood of $K$ containing $U$. Another way to characterize attractors (see [2, Theorem 2.13., p. 73] for a precise statement) is by the existence of Lyapunov functions. A Lyapunov function for $K$ is a continuous $\Phi: \mathcal{A}(K) \longrightarrow[0,+\infty)$ such that (1) $\left.\Phi\right|_{K} \equiv 0,(2) \Phi$ is strictly decreasing along trajectories in $\mathcal{A}(K)-K$ (explicitly, $\Phi(x \cdot t)<\Phi(x)$ for any $x \in \mathcal{A}(K)-K$ and $t>0$ ); finally (3) $\Phi$ is uniformly unbounded (this means that for every $R \geq 0$ there exists a compact set $P_{R} \subseteq \mathcal{A}(K)$ such that $\left.\Phi(x) \geq R \forall x \notin P_{R}\right)$. We shall explictly state a useful consequence of (3), namely (4) the sets $\{x \in \mathcal{A}(K): \Phi(x) \leq r\}$ are compact for every $r \geq 0$. Dual notions are obtained by reversing the flow (repellers and Lyapunov functions for repellers). A good reference for this elementary theory is 2 .

Following Conley we shall deal only with isolated compact invariant sets. These are compact invariant sets $K$ which posess a so-called isolating neighbourhood, that is, a compact neighbourhood $N$ such that $K$ is the maximal invariant set in $N$, or setting

$$
N^{+}:=\{x \in N: x \cdot[0,+\infty) \subseteq N\} \text { and } N^{-}:=\{x \in N: x \cdot(-\infty, 0] \subseteq N\}
$$

such that $K=N^{+} \cap N^{-}$. An isolating block is an isolating neighbourhood which has special topological properties. More precisely, an isolating block $N$ is an isolating neighbourhood such that there are compact sets $N^{i}, N^{o} \subseteq \partial N$, called the entrance and exit sets, satisfying

(1) $\partial N=N^{i} \cup N^{o}$,

(2) for every $x \in N^{i}$ there exists $\varepsilon>0$ such that $x \cdot[-\varepsilon, 0) \subseteq M-N$ and for every $x \in N^{o}$ there exists $\delta>0$ such that $x \cdot(0, \delta] \subseteq M-N$, 
(3) for every $x \in \partial N-N^{i}$ there exists $\varepsilon>0$ such that $x \cdot[-\varepsilon, 0) \subseteq$ int $N$ and for every $x \in \partial N-N^{o}$ there exists $\delta>0$ such that $x \cdot(0, \delta] \subseteq$ int $N$.

These blocks form a neighbourhood basis of $K$ in $M$ (see [5] or [7]) and consitute an operative means of calculating the homotopical Conley index $h(K)$ of $K$, which is defined as the homotopy type of the pointed pair $\left(N / N^{o},\left[N^{o}\right]\right)$. Quite a lot of effort is required to prove that this definition is correct or to derive its essential properties, so we refer the reader either to the foundational monography by Conley [6] or the more recent book [9. Since the homotopy index $h(K)$ is not suitable for calculations, we shall deal here with the cohomological index $C H^{*}(K)$, defined simply as the cohomology graded module $H^{*}\left(N / N^{o},\left[N^{o}\right]\right)$. This module agrees with $H^{*}\left(N, N^{o}\right)$ when Cech theory is used.

As for the algebraic topology part, the book 21] is a good reference. Let us note here that whenever cohomology or homology are used, reduced Čech theory is intended. This theory agrees with the singular one when the underlying space is an ANR (for information on ANR's, see [13]). Finally, the essentials of shape theory are contained in [4] or, for more exhaustive information, 8] and [15].

\section{ON The SHAPE OF $D$}

We start off by investigating what can be said about the shape of the invariant set $D=\partial \mathcal{A}(K)$, the boundary of the basin of attraction of an attractor $K$. The simplest situation one can imagine is that of $K$ being an attracting equilibrium point in $\mathbb{R}^{2}$ or in $\mathbb{R}^{3}$, but even with such a good picture in mind things can be not easy at all. The following examples show this.

Example 2.1. Consider the case depicted in Figure 1 below. An attracting point $p$ is shown whose basin of attraction $\mathcal{A}(p)$ (the shaded region in the drawing) is homeomorphic to an open disk, however its boundary $D=\partial \mathcal{A}(p)$ does not have polyhedral shape. It should be understood that the boundary $D$ consists of fixed points and is self-tangent infinitely many times, so that it cuts out infinitely many holes in $\mathbb{R}^{2}$; therefore it cannot have polyhedral shape because, by Alexander's duality, $\check{H}^{1}(D)$ is not finitely generated.

Example 2.2. A similar situation (shown in Figure 2) can happen in $\mathbb{R}^{3}$. Again, the attractor consists of one point $p$ (not shown) with $\mathcal{A}(p)$ the shaded region, which is homeomorphic to an open 3-cell. However, the boundary $D=\partial \mathcal{A}(p)$ does not have polyhedral shape by the same reason as above.

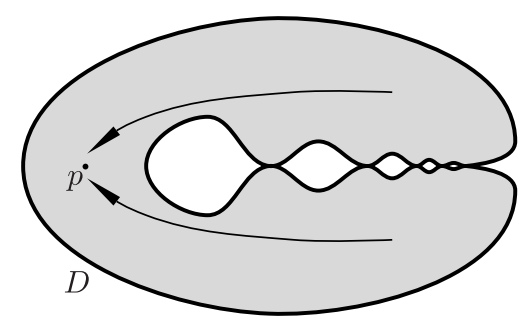

Figure 1. An attracting point $p$ in $\mathbb{R}^{2}$ (note that $\overline{\mathcal{A}(p)}$ is a disk with infinitely many holes). 


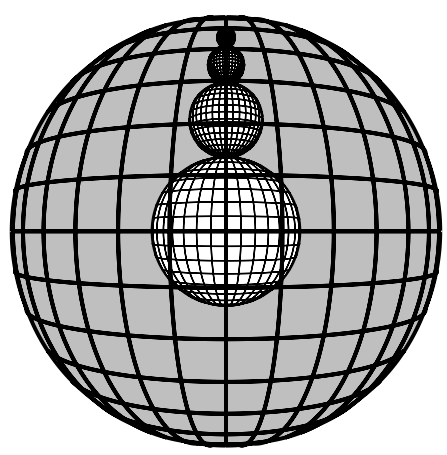

FIgURE 2. A similar situation in $\mathbb{R}^{3}$.

The inclusion $i: K \hookrightarrow \mathcal{A}(K)$ is a shape equivalence and, when the phase space is an ANR, $K$ has the shape of a finite polyhedron and so does $\mathcal{A}(K)$. Therefore both have a finite number of connected components. This property transfers nicely onto $D$.

Proposition 2.3. Let $K \subseteq M$ be an attractor in the locally compact ANR $M$ and assume the boundary $D=\partial \mathcal{A}(K)$ of the basin of attraction of $K$ is compact. Then it has finitely many connected components.

Proof. Choose a Lyapunov function $\Phi: \mathcal{A}(K) \longrightarrow[0,+\infty)$ and consider the sets $U \supseteq \Sigma$ defined by $U=\{x \in \mathcal{A}(K): 0<\Phi(x)<2\}$ and $\Sigma=\{x \in \mathcal{A}(K): \Phi(x)=$ $1\}$. It is easy to see that the inclusion $i: \Sigma \hookrightarrow U$ is a homotopy equivalence. Now $U$ is an ANR (because it is open in $M$, which is an ANR itself), hence so is the compact set $\Sigma$ (being a retract of an ANR), and therefore it has a finite number of connected components, which we shall call $\Sigma_{1}, \ldots, \Sigma_{n}$. The proof will be finished if we show that $D=\alpha(\Sigma)$, since then $D=\alpha(\Sigma)=\alpha\left(\Sigma_{1}\right) \cup \ldots \cup \alpha\left(\Sigma_{n}\right)$ where each $\alpha\left(\Sigma_{k}\right)$ is connected, hence $D$ has no more than $n$ components. To establish the equality $D=\alpha(\Sigma)$ only the inclusion $D \subseteq \alpha(\Sigma)$ needs some argument, because the reverse one follows inmediately from the fact that $K$ is an attractor. So pick a point $x \in D$ and a sequence $\left(x_{k}\right)_{k \in \mathbb{N}} \subseteq \mathcal{A}(K)-K$ converging to $x$. Since $\Sigma$ is a section of $\mathcal{A}(K)-K$, there exists a unique sequence $\left(t_{k}\right)_{k \in \mathbb{N}} \subseteq \mathbb{R}$ such that $\sigma_{k}=x_{k} \cdot t_{k} \in \Sigma$ for every $k \in \mathbb{N}$. It is clear that $t_{k} \rightarrow+\infty$, therefore $x=\lim \sigma_{k} \cdot\left(-t_{k}\right) \in \alpha(\Sigma)$.

The previous examples share the feature that $D$ does not have polyhedral shape because it cuts out infinitely many holes in $\mathbb{R}^{2}$ or $\mathbb{R}^{3}$, but this also means that $D$ is not an isolated set, so in some sense it is unsuitable for dynamical investigation with tools such as Conley's index. However, consider the following example.

Example 2.4. The flow depicted in Figure 3 shows an attracting point $p$ such that the boundary of its basin of attraction is an isolated set but does not have polyhedral shape (hence $D$ being isolated is still not enough to obtain good topological properties). To be precise, let $Q$ be the closure of the basin of attraction of the point $p$ in Example 2.1 and assume it to be embedded in $\mathbb{R}^{2} \times\{0\} \subseteq \mathbb{R}^{3}$. Take its cartesian product by the interval $[-1,1]$ to obtain a cylinder $C=Q \times[-1,1]$ with infinitely many cylinders removed, corresponding to the holes in $Q$. Now the boundary $D$ of this cylinder is formed by the cartesian product $\partial Q \times[-1,1]$ together with the upper and lower lids of $C$, that is, $Q \times\{-1,1\}$. Let every point 


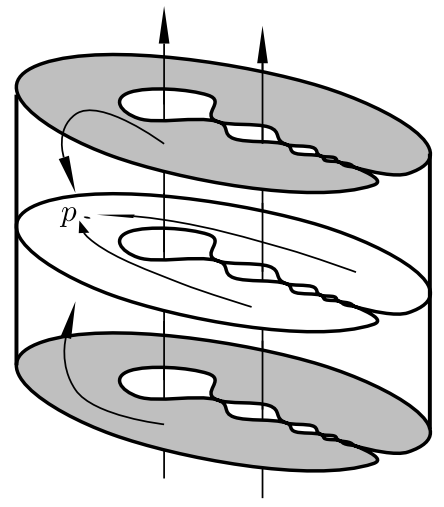

FiguRE 3. A nonpolyhedral shape boundary.

in $D$ be fixed and any other in $C$ approach $p$ so that it is an attractor. Otherwise (that is, outside $C$ ), let the flow lines be parallel to the $z$-axis and move upwards.

The construction explained in the example above works equally well if we start with a compact closure $Q$ of any basin of attraction in $\mathbb{R}^{n}$ whose boundary is stationary under the flow and does not have polyhedral shape, so in fact there exist big families of examples which make it clear that for the result in Proposition 2.3 to be improved further conditions on $D$ have to be imposed. In Theorem 2.6 we exemplify this by means of a Conley index condition. We begin with a lemma which recalls the by now classical technique to be used in the proof of the theorem.

Lemma 2.5. Let $K$ be a compact invariant set in a locally compact ANR $M$. Suppose $K$ has an isolating neighbourhood $N$ such that $N=N^{+} \cup N^{-}$. Then $K$ has the shape of a finite polyhedron.

Proof. We will just give an outline of the proof because the argument involved is standard. First of all observe that $K$ attracts $N^{+}$and repells $N^{-}$. Indeed, for any $x \in N^{+}$the relation $x \cdot[0,+\infty) \subseteq N$ implies that $\emptyset \neq \omega(x) \subseteq N$ and, since $\omega(x)$ is a nonempty invariant set contained in $N$ (which isolates $K$ ) the inclusion $\omega(x) \subseteq K$ must hold. Now there exists a decreasing sequence of compact neighbourhoods $\left\{Q_{k}\right\}_{k \in \mathbb{N}}$ which form a neighbourhood basis of $K$ such that (1) $Q_{1}$ is a compact ANR and (2) every inclusion map $i_{k}: Q_{k+1} \hookrightarrow Q_{k}$ is a homotopy equivalence. These are constructed pasting together positively (resp. negatively) invariant neighbourhoods of $K$ in $N^{+}$(resp. $N^{-}$). Property (1) is inherited from the phase space because $Q_{1}$ can be chosen so as to be a retract of int $(N)$ (as in the proof of Proposition 2.3) and for property (2) a homotopy inverse for $i_{k}$ is provided by the flow (see, for example, 20, Lemma 2., p. 523]). All these facts together with a result of West ([22]) prove that $K$ has the shape of a finite polyhedron.

Theorem 2.6. Let $K \subseteq M$ be an attractor in the locally compact $A N R M$ and suppose that the boundary $D$ of the basin of attraction $\mathcal{A}(K)$ of $K$ is an isolated compactum whose 1-dimensional cohomological Conley index satisfies $C \check{H}^{1}(D)=0$. Then $D$ has the shape of a finite polyhedron.

Proof. Step 1. $D$ has a positively invariant isolating neighbourhood $P$ in $M-\mathcal{A}(K)$. 
Proof. Let $D=D_{1} \cup \ldots \cup D_{n}$ be the (finitely many by Proposition 2.3) connected components of $D$, which are themselves isolated compacta. For each $1 \leq k \leq n$ there exists a connected isolating block $\left(N_{k}, N_{k}^{i}, N_{k}^{o}\right)$ for $D_{k}$ such that $N_{k} \cap N_{\ell}=\emptyset$ if $k \neq \ell$ (since every $D_{k}$ has a neighbourhood basis of connected isolating blocks). Observe that, by the additivity of Conley's index, $0=C \check{H}^{1}(D) \cong \bigoplus_{k=1}^{n} C \check{H}^{1}\left(D_{k}\right)$ and so $C \check{H}^{1}\left(D_{k}\right)=0 \forall 1 \leq k \leq n$. Now let us prove that every $N_{k}-\mathcal{A}(K)$ is positively invariant, and so $P=\left(N_{1} \cup \ldots \cup N_{n}\right)-\mathcal{A}(K)$ will do the job. From the exact sequence in reduced Čech cohomology

$$
0 \rightarrow \check{H}^{0}\left(N_{k}, N_{k}^{o}\right) \rightarrow \check{H}^{0}\left(N_{k}\right) \rightarrow \check{H}^{0}\left(N_{k}^{o}\right) \rightarrow \check{H}^{1}\left(N_{k}, N_{k}^{o}\right) \rightarrow \ldots
$$

we see that $N_{k}^{o}$ is connected because $\check{H}^{0}\left(N_{k}\right)=0\left(N_{k}\right.$ is connected $), \check{H}^{1}\left(N_{k}, N_{k}^{o}\right) \cong$ $C \check{H}^{1}\left(D_{k}\right)=0$ by hypothesis and therefore $\check{H}^{0}\left(N_{k}^{o}\right)=0$. Now observe that $\emptyset \neq$ $D_{k} \subseteq$ int $N_{k} \cap \partial \mathcal{A}(K)$ so int $N_{k} \cap \mathcal{A}(K) \neq \emptyset$ and there exist $x \in N_{k} \cap \mathcal{A}(K)$ and $t>0$ such that $x \cdot t \notin N_{k}$ (otherwise, $x \cdot[0,+\infty) \subseteq N_{k}$ and $\emptyset \neq \omega(x) \subseteq N_{k}$ which is impossible as $\omega(x) \subseteq K$, but $N_{k}$ isolates $D_{k}$, disjoint from $\left.K\right)$. Hence the positive semitrajectory $\gamma^{+}(x) \subseteq \mathcal{A}(K)$ of $x$ must meet the exit set $N_{k}^{o}$, and it follows that $N_{k}^{o} \cap \mathcal{A}(K) \neq \emptyset$. Moreover, since $N_{k}^{o} \cap D=\left(N_{k}^{o} \cap D_{1}\right) \cup \ldots \cup\left(N_{k}^{o} \cap D_{n}\right) \subseteq$ $\left(N_{k} \cap N_{1}\right) \cup \ldots \cup\left(N_{k}^{o} \cap D_{k}\right) \cup \ldots \cup\left(N_{k} \cap N_{n}\right)=\emptyset$ we get that the connected set $N_{k}^{o}$ lies in the disjoint union of the two open sets $\mathcal{A}(K)$ and $M-\overline{\mathcal{A}(K)}$, so in fact $N_{k}^{o} \subseteq \mathcal{A}(K)$. Then $N_{k}-\mathcal{A}(K)$ is positively invariant because for any $x \in N_{k}-\mathcal{A}(K)$ we have $\gamma^{+}(x) \subseteq M-\mathcal{A}(K)$, and this prevents $x$ from exiting $N_{k}$ since we proved that $N_{k}^{o} \subseteq \mathcal{A}(K)$.

Step 2. $D$ has a negatively invariant isolating neighbourhood $Q$ in $\overline{\mathcal{A}(K)}$.

Proof. Let us consider the one-point compactification $M_{\infty}$ of $M$ obtained by adjoining the point $\infty$ to $M$, and extend the flow letting $\{\infty\}$ be a fixed point. Observe that $K$ is still an attractor with the same region of attraction $\mathcal{A}(K)$, and call $D_{\infty}:=\partial_{M_{\infty}} \mathcal{A}(K)$ the boundary of $\mathcal{A}(K)$ in $M_{\infty}$. It is clear that $D_{\infty}=D$ if $\overline{\mathcal{A}(K)}$ (the closure of $\mathcal{A}(K)$ in $M$ ) is compact or $D_{\infty}=D \cup\{\infty\}$ if it is not. Now pick a Lyapunov function $\Phi: \mathcal{A}(K) \longrightarrow[0,+\infty)$ for the attractor $K$ and extend it to $\overline{\mathcal{A}(K)}^{M_{\infty}}$ (the closure of $\mathcal{A}(K)$ in $M_{\infty}$ ) by letting it assume the value $+\infty$ on $D_{\infty}$ (this extension is continuous because of the uniform unboundedness of $\Phi$ ).

For every $n \in \mathbb{N}$ the set $Q_{n}=\left\{x \in \overline{\mathcal{A}(K)}^{M_{\infty}}: \Phi(x) \geq n\right\}$ is a negatively invariant isolating neighbourhood of $D_{\infty}$ in $\overline{\mathcal{A}(K)}^{M_{\infty}}$. If $\overline{\mathcal{A}(K)}$ is compact we are done: $Q=Q_{1}$ serves our purpose because $\overline{\mathcal{A}(K)}$ agrees with $\overline{\mathcal{A}(K)}^{M_{\infty}}$. Otherwise, choose disjoint open sets $U_{\infty} \supseteq\{\infty\}$ and $U_{D} \supseteq D$ and an index $n_{0} \in \mathbb{N}$ such that $Q_{n_{0}} \subseteq U_{\infty} \cup U_{D}$ (this is possible, since $\left\{Q_{n}\right\}_{n \in \mathbb{N}}$ is a decreasing neighbourhood basis of $D_{\infty}=D \cup\{\infty\}$ and $D$ was assumed to be compact). Letting $Q=Q_{n_{0}} \cap U_{D}=$ $Q_{n_{0}} \cap\left(M_{\infty}-U_{\infty}\right)$, it is easy to see that $Q$ is a negatively invariant isolating neighbourhood of $D$ in $\overline{\mathcal{A}(K)}$.

Step 3. $N=P \cup Q$ is an isolating neighbourhood of $D$ in $M$ such that $N=$ $N^{+} \cup N^{-}$. Therefore, by Lemma 2.5. $N$ has the shape of a finite polyhedron.

Proof. It is clear that $N^{+}=P$ and $N^{-}=Q$, so the equality $N=N^{+} \cup N^{-}$ follows at once. Hence it will be enough to show that $N$ is indeed a neighbourhood of $D$. Since $P$ and $Q$ are neighbourhoods of $D$ in $M-\mathcal{A}(K)$ and $\overline{\mathcal{A}(K)}$, respectively, there exist open sets $M \supseteq U \supseteq D$ and $M \supseteq V \supseteq D$ such that $P \supseteq U-\mathcal{A}(K)$ and $Q \supseteq V \cap \overline{\mathcal{A}(K)}$. Now $U \cap V$ is an open set which contains $D$ and is contained in 
$N$, because if $x \in U \cap V$ but $x \notin P$, then $x \in \mathcal{A}(K)$ since $P \supseteq U-\mathcal{A}(K)$ and so $x \in V \cap \mathcal{A}(K) \subseteq Q$.

Remark 2.7. Let us compute the 1-dimensional Conley index for Example2.4. Pick, for example, a ball $B \supseteq C$ and a small ball $B^{\prime}$ around $p$ so that $N=B-\operatorname{int} B^{\prime}$ is an isolating neighbourhood for $D$. If $B$ and $B^{\prime}$ are reasonably chosen, $N$ will be an isolating block for $D$ whose exit set $L$ consists of the upper hemisphere of $\partial B$ and the whole of $\partial B^{\prime}$. Thus the quotient $(N / L, L / L)$ has the same homotopy type as a pointed $\mathbb{S}^{1}$ and $C \check{H}^{1}(D) \cong \mathbb{Z}$ (it is nonzero, as we expected by Theorem 2.6).

\section{From local to global: The dynamics in $M$}

This section will be devoted to showing how the condition on the Conley index of $D$ which was considered above not only renders shape theoretic results, but in fact allows us to have a global picture of the dynamics in $M$ (see Proposition 3.3) which is especially precise when $M$ is low-dimensional, for example when $M=\mathbb{S}^{3}$ (Corollary 3.6 and Remark 3.7). The starting point for this analysis is the proof of Theorem 2.6. where one can observe that the key is that $D$ repells points in $\mathcal{A}(K)-K$ and attracts points outside $\overline{\mathcal{A}(K)}$ (and this dynamical behaviour is deduced from the hypothesis on the Conley index of $D$ ). Therefore it is only natural to believe that $\overline{\mathcal{A}(K)}$ is an attractor in $M$, and this is the case provided it is compact.

Remark 3.1. Let $K$ be an attractor in a locally compact ANR $M$. Assume that the hypotheses in Theorem 2.6 hold and, in addition, $\overline{\mathcal{A}(K)}$ is compact. Then $\overline{\mathcal{A}(K)}$ is an attractor in $M$.

To prove this, let $N=\overline{\mathcal{A}(K)} \cup P$, where $P$ is a positively invariant neighbourhood of $D$ in $M-\mathcal{A}(K)$ (it was established in Step 1 of Theorem 2.6 that such a neighbourhood exists). It is clear that $N$ is a compact neighbourhood of $\overline{\mathcal{A}(K)}$ and, since it is the union of two positively invariant sets, $N$ is also positively invariant. It only remains to check that $N$ isolates $\overline{\mathcal{A}(K)}$, but this is easy.

Example 3.2. The condition that $\overline{\mathcal{A}(K)}$ be compact, despite its topological nature, has some dynamical content: Consider the flow in the real line depicted in Figure [4 (a), where 0 is an attractor whose region of attraction is the interval $\mathcal{A}(0)=$ $(-\infty, 1)$ and otherwise points in $(1,+\infty)$ approach 1 . Here $D=\partial \mathcal{A}(0)=\{1\}$ and $C \check{H}^{1}(D)=0$, so we are in the hypotheses of Theorem 2.6 (indeed $D$ has polyhedral shape). Now, it does not make sense to say that $\overline{\mathcal{A}(0)}=(-\infty, 1]$ is an attractor in $\mathbb{R}$, since it is not compact. Thus one can compactify the phase space to obtain a flow in $\mathbb{S}^{1}$, where the point at infinity is fixed (Figure $4(b)$ ). Now it is clear that $\overline{\mathcal{A}(0)}^{\mathbb{S}^{1}}$ is not an attractor.

A reasonable way to ensure that $\overline{\mathcal{A}(K)}$ is compact is to focus our attention on compact phase spaces $M$, and we will do so in the sequel. On the other hand, we no longer need the hypothesis that $M$ be an ANR (this was used only in the final step of Theorem 2.6). Under these assumptions, the following proposition gives a fairly clear picture of the situation.

Proposition 3.3. Let $K$ be an attractor in a compact phase space $M$. Assume that $D=\partial \mathcal{A}(K)$ is an isolated set whose 1-dimensional cohomological Conley index is zero. Then there exists a repeller $K^{\prime}$ in $M$ such that the boundary of $\mathcal{R}\left(K^{\prime}\right)$ coincides with $D$ and $M=\mathcal{A}(K) \cup D \cup \mathcal{R}\left(K^{\prime}\right)$, where the union is disjoint. 


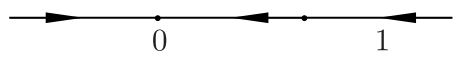

(a)

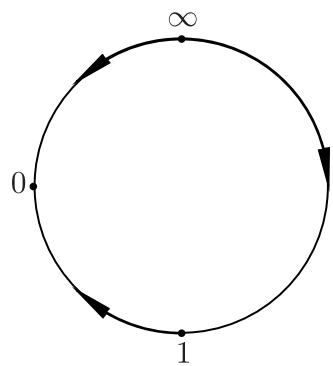

(b)

Figure 4 . The compactness of $\overline{\mathcal{A}(K)}$ is necessary.

Proof. By virtue of Remark 3.1, the compact set $\overline{\mathcal{A}(K)}$ is an attractor in $M$, therefore it has a dual repeller $K^{\prime}$. We recall here that $K^{\prime}=\{x \in M: \omega(x) \cap \overline{\mathcal{A}(K)}=\emptyset\}$, and it agrees with the set of points in $M$ which are not attracted by $\overline{\mathcal{A}(K)}$ (see [18] for an exposition of these results). Now $\left\{K^{\prime}, \overline{\mathcal{A}(K)}\right\}$ is a Morse decomposition of $M$ ([18, Lemma 3.2. and Proposition 3.4.iv]) and therefore $\mathcal{R}\left(K^{\prime}\right)=M-\overline{\mathcal{A}(K)}$; hence $\partial \mathcal{R}\left(K^{\prime}\right)=\partial \overline{\mathcal{A}(K)}=\partial \mathcal{A}(K)=D$. To finish the proof we shall observe that $\mathcal{A}(K) \cap \mathcal{R}\left(K^{\prime}\right)=\emptyset$. For, if $x \in \mathcal{A}(K) \cap \mathcal{R}\left(K^{\prime}\right)$, then $\emptyset \neq \alpha(x) \subseteq D$ (since $x \in \mathcal{A}(K) \subseteq \overline{\mathcal{A}(K)}$ and this set is compact and invariant) but, on the other hand, $\alpha(x) \subseteq K^{\prime}$ (because $x \in \mathcal{R}\left(K^{\prime}\right)$ ). Therefore $D \cap K^{\prime} \supseteq \alpha(x) \neq \emptyset$, contradicting the fact that $D$ is the boundary of $\mathcal{R}\left(K^{\prime}\right)$, which is a neighbourhood of $K^{\prime}$ in $M$.

The scenario just described (that is, $M$ being the union of a basin of attraction $\mathcal{A}(K)$ and a basin of repulsion $\mathcal{R}\left(K^{\prime}\right)$ with a common boundary $D$, these three sets must be disjoint by the same argument as in Proposition 3.3. takes place when $C \check{H}^{1}(D)=0$, although in general this is by no means a necessary condition, as shown by the following example.

Example 3.4. The phase space is $\mathbb{S}^{3}$, best thought of as $\mathbb{R}^{3}$ together with the point at infinity. Take the unit ball $B$ with its origin $p$ being an attractor in $B$ (every point in the boundary is fixed) and nip two little horns until they become tangent to each other, as depicted in Figure 5. Then the basin of attraction $\mathcal{A}(p)$ is an open ball and its closure is the horned ball, which has the same homotopy type as a solid torus. Here its complement (in $\mathbb{S}^{3}$ ) is another torus and the dual repeller of $p$ is the core of this torus, a circumference $K^{\prime}$. However $C \breve{H}^{1}(D)=\mathbb{Z}$ (this can be checked either by direct computation or using Proposition 3.5 below).

Therefore the condition $C \check{H}^{1}(D)=0$ is sufficient, but not necessary, for the phase space to have a decomposition as the union of the basins of an attractor $K$, a repeller $K^{\prime}$ and their common boundary $D$. Still, the Conley index of $D$ plays an important role in this situation: clearly the example above shows that $K$ does not determine $K^{\prime}$, because for the same $K$ (a singleton) the construction can be easily modified so that $K^{\prime}$ is a bouquet of any (finite) number of circumferences, but it turns out that information about $K$ plus some knowledge of the Conley index of $D$ can determine $K^{\prime}$ up to homology (and vice versa). Proposition 3.5 and its corollary are significant instances of this. 


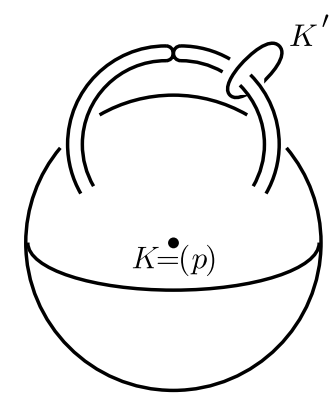

FIGURE 5. An attracting point and its dual repelling circumference.

Proposition 3.5. Let $K, K^{\prime} \subseteq \mathbb{S}^{n}$ be, respectively, an attractor and repeller whose basins $\mathcal{A}(K)$ and $\mathcal{R}\left(K^{\prime}\right)$ have a common boundary $D$ and such that $\mathbb{S}^{n}=\mathcal{A}(K) \cup$ $D \cup \mathcal{R}\left(K^{\prime}\right)$. If $K$ has trivial shape, then $\check{H}_{k}\left(K^{\prime}\right) \cong C H^{n-k-1}(D)$.

Proof. Let $\Phi: \overline{\mathcal{A}(K)} \longrightarrow[0,+\infty]$ and $\Psi: \overline{\mathcal{R}\left(K^{\prime}\right)} \longrightarrow[0,+\infty]$ be Lyapunov functions for $K$ and $K^{\prime}$ extended as $+\infty$ to the boundary of the basins of attraction and repulsion, respectively. Since their domains intersect only in $D$ (recall that $\mathcal{A}(K) \cap \mathcal{R}\left(K^{\prime}\right)=\emptyset$ necessarily), where they both assume the value $+\infty$, they can be pasted together to yield a well defined continuous function $H: \mathbb{S}^{n} \longrightarrow[0,+\infty]$ with the following properties: (1) $H^{-1}(+\infty)=D,(2) H^{-1}(0)=K \cup K^{\prime}$ and (3) $H$ is decreasing on trajectories contained in $\mathcal{A}(K)-K$ and increasing on trajectories contained in $\mathcal{R}\left(K^{\prime}\right)-K^{\prime}$.

To compute the Conley index for $D$ we shall choose an adequate isolating block, to be described now. Set $N=\left\{x \in \mathbb{S}^{n}: H(x) \geq 1\right\}$, which is a compact neighbourhood of $D$ because $H$ is continuous. Property (3) above ensures that $N$ is an isolating block of $D$ whose exit set is $N^{o}=\{x \in \mathcal{A}(K): H(x)=1\}=\{x \in \mathcal{A}(K)$ : $\Phi(x)=1\}$.

Finally, let $U_{1}=\{x \in \mathcal{A}(K): H(x)<1\}, U_{2}=\{x \in \mathcal{A}(K): H(x)<2\}$ and $U_{1}^{\prime}=\left\{x \in \mathcal{R}\left(K^{\prime}\right): H(x)<1\right\}$. Observe that the inclusions $K \hookrightarrow U_{1} \hookrightarrow$ $U_{2}$ and $K^{\prime} \hookrightarrow U_{1}^{\prime}$ are all shape equivalences since $U_{1}, U_{1}^{\prime}$ are positively invariant neighbourhoods of $K$ and $U_{1}^{\prime}$ is a negatively invariant neighbourhood of $K^{\prime}$. Hence $U_{1}$ and $U_{2}$ have trivial shape.

Now we can proceed to the computations: we have that $\check{H}_{k}\left(K^{\prime}\right) \cong \check{H}_{k}\left(U_{1}^{\prime}\right)=$ $\check{H}_{k}\left(\mathbb{S}^{n}-\left(U_{2} \cup N\right)\right) \cong \check{H}^{n-k-1}\left(U_{2} \cup N\right)$ by the fact that $K^{\prime} \hookrightarrow U_{1}^{\prime}$ is a shape equivalence and by Alexander's duality. Since $U_{2}$ has trivial shape, $\check{H}^{n-k-1}\left(U_{2} \cup\right.$ $N) \cong \check{H}^{n-k-1}\left(U_{2} \cup N, U_{2}\right) \cong \check{H}^{n-k-1}\left(N, U_{2}-U_{1}\right)$ by excision. $U_{2}-U_{1}$ deformation retracts onto $N^{o}$; therefore, $\check{H}_{k}\left(K^{\prime}\right) \cong \check{H}^{n-k-1}\left(N, N^{o}\right) \cong C \check{H}^{n-k-1}(D)$.

Corollary 3.6. Let $K \subseteq \mathbb{S}^{3}$ be an attractor with trivial shape and suppose that the boundary $D$ of its basin of attraction satisfies $C \check{H}^{1}(D)=0$. Then the dual repeller $K^{\prime}$ of $\overline{\mathcal{A}(K)}$ has a finite number of components, each of which has trivial shape. In particular, if $C \check{H}^{2}(D)=0$, then $K^{\prime}$ has trivial shape.

Proof. We begin by checking that the homology groups of every component of $K^{\prime}$ are trivial. By Proposition 3.5 and hypothesis, we have $\check{H}_{1}\left(K^{\prime}\right) \cong C \check{H}^{1}(D)=0$. Next, from the exact sequence in Step 1 of the proof of Theorem 2.6 it follows that $C \check{H}^{0}(D)=0$; therefore (again by Proposition 3.5 ), $\check{H}_{2}\left(K^{\prime}\right) \cong C \check{H}^{0}(D)=0$. 
Finally, if $C^{\prime}$ is a component of $K^{\prime}$ (of which there are only a finite amount since $K^{\prime}$ is a repeller in an ANR), its homology groups are direct summands of those of $K^{\prime}$, hence trivial too.

By virtue of [14, Theorem 4.5., p. 181] it only remains to check that $C^{\prime}$ is pointed 1-movable. This we do as follows: first of all, since $C^{\prime}$ has the shape of a finite polyhedron, it is a FANR ([15, Theorem 14., p. 234]). Now for any choice of a basepoint $*$ in $C^{\prime}$, the pointed space $\left(C^{\prime}, *\right)$ is also a FANR (this is 15, Theorem 19., p. 236], due to Hastings and Heller). Finally, every pointed FANR is pointed movable ([15, Theorem 18., p. 235]). Therefore $\left(C^{\prime}, *\right)$ is pointed 1-movable and the result follows.

The last assertion follows again from Proposition 3.5, since $\check{H}_{0}\left(K^{\prime}\right) \cong C \check{H}^{2}(D)$. Hence if the latter group is trivial, $K^{\prime}$ is connected and has trivial shape.

Remark 3.7. A reciprocal statement for Corollary 3.6 holds true: if $K \subseteq \mathbb{S}^{3}$ is an attractor with trivial shape and there exists a repeller $K^{\prime} \subseteq \mathbb{S}^{3}$ such that $\mathbb{S}^{3}=$ $\mathcal{A}(K) \cup D \cup \mathcal{R}\left(K^{\prime}\right)$ and every component of $K^{\prime}$ has trivial shape, then $C \check{H}^{1}(D)=0$. This follows immediately from Proposition 3.5 and shows how a Conley-index type condition on $D$ can be enough, under favourable hypotheses, to characterize the dynamics for an ample family of dynamical systems in $\mathbb{S}^{3}$.

The essential tool in Proposition 3.5 was Alexander's duality theorem. In case the flow is differentiable, there exist isolating blocks which are manifolds and so other forms of duality are available, such as Lefschetz's duality. These also render some results in our context.

Proposition 3.8. Let $K$ be an attractor for a differentiable flow in an orientable compact n-manifold $M$. Then the number of connected components of the dual repeller $K^{\prime}$ of $\overline{\mathcal{A}(K)}$ is bounded above by $\mathrm{rk} C \check{H}^{n-1}(D)+\#$ (components of $\left.D\right)$.

Proof. Proceed as in Proposition 3.5 to obtain a function $H: M \longrightarrow[0,+\infty]$ with the same properties as above. Since the flow is differentiable, the Lyapunov functions used to construct $H$ can be chosen to be differentiable and so will be $H$, except (possibly) on $D$. However by Sard's Theorem ([16, Corollary, p. 11]) the restriction $\left.H\right|_{M-D}$ has a regular value and (see, for example, 16, Lemma 3., p. 12]) it is clear that for some real number $R>0$ the set $N=\{x \in M: H(x) \geq R\}$ is a compact $n$-manifold with boundary $\partial N=\{x \in M: H(x)=R\}$. Observe furthermore that $\partial N$ is the disjoint union of $N^{o}=\{x \in \mathcal{A}(K): H(x)=R\}$ and $N^{i}=\left\{x \in \mathcal{R}\left(K^{\prime}\right): H(x)=R\right\}$.

Let $K^{\prime}=K_{1}^{\prime} \cup \ldots \cup K_{m}^{\prime}$ be the components of $K^{\prime}$ (these are finite in number since $K^{\prime}$ has the shape of a finite polyhedron). Each one is a repeller by itself (this is standard, see [2]) with its own region of repulsion, and $\mathcal{R}\left(K^{\prime}\right)=\mathcal{R}\left(K_{1}^{\prime}\right) \cup \ldots \cup$ $\mathcal{R}\left(K_{m}^{\prime}\right)$ is the disjoint union of them. Therefore $N^{i}$, which must intersect every $\mathcal{R}\left(K_{i}^{\prime}\right)$, has at least $m$ components. That is, $m \leq \mathrm{rk} \check{H}_{0}\left(N^{i}\right)$, where unreduced homology is used to avoid complications in notation.

Consider the following exact sequence in unreduced Čech homology:

$$
\ldots \rightarrow \check{H}_{1}\left(N, N^{i}\right) \rightarrow \check{H}_{0}\left(N^{i}\right) \rightarrow \check{H}_{0}(N) \rightarrow \check{H}_{0}\left(N, N^{i}\right) \rightarrow 0
$$

which gives rise to the inequality rk $\check{H}_{0}\left(N^{i}\right)+\operatorname{rk} \check{H}_{0}\left(N, N^{i}\right) \leq \operatorname{rk} \check{H}_{1}\left(N, N^{i}\right)+$ rk $\check{H}_{0}(N)$. Now $\check{H}_{0}\left(N, N^{i}\right)=0$ (the reason is the same as in Corollary 3.6), the rank of $\breve{H}_{0}(N)$ is the number of components of $N, m \leq \mathrm{rk} \check{H}_{0}\left(N^{i}\right)$ and (by Lefschetz duality) $\check{H}_{1}\left(N, N^{i}\right) \cong \check{H}^{n-1}\left(N, N^{o}\right) \cong C \check{H}^{n-1}(D)$. Therefore substituting in the 
inequality we get $m \leq \operatorname{rk} C \check{H}^{n-1}(D)+\#($ components of $N)$. We finish checking that $N$ has the same number of components as $D$, and this is due to the fact that every component of $D$ is contained in one of $N$ (which is clear) and every component of $N$ cuts $D$. Indeed, let $N^{*}$ be a component of $N$ and pick $x \in N^{*}$; then either $x \cdot[0,+\infty) \subseteq N^{*}$ or $x \cdot(-\infty, 0] \subseteq N^{*}$, which in either case implies that $\emptyset \neq \omega(x) \subseteq D \cap N^{*}$ or $\emptyset \neq \alpha(x) \subseteq D \cap N^{*}$.

If the phase space $M$ is not orientable, then the preceding argument goes through using coefficients in $\mathbb{Z}_{2}$.

\section{REFERENCES}

1. J. Auslander, N. P. Bhatia, and P. Seibert, Attractors in dynamical systems, Bol. Soc. Mat. Mexicana (2) 9 (1964), 55-66. MR0188535 (32:5973)

2. N. P. Bhatia and G. P. Szegö, Stability theory of dynamical systems, Die Grundlehren der mathematischen Wissenschaften, Band 161, Springer-Verlag, 1970. MR0289890(44:7077)

3. S. A. Bogatyı̆ and V. I. Gutsu, On the structure of attracting compacta, Differentsialnye Uravneniya 25 (1989), no. 5, 907-909, 920. MR1003051 (90j:58076)

4. K. Borsuk, Theory of shape, Monografie Matematyczne, Tom 59, Państwowe Wydawnictwo Naukowe, 1975. MR0418088 (54:6132)

5. R. C. Churchill, Isolated invariant sets in compact metric spaces, J. Diff. Eq. 12 (1972), 330-352. MR0336763 (49:1536)

6. C. Conley, Isolated invariant sets and the Morse index, CBMS Regional Conference Series in Mathematics, 38, American Mathematical Society, 1978. MR511133 (80c:58009)

7. C. Conley and R. Easton, Isolated invariant sets and isolating blocks, Trans. Amer. Math. Soc. 158 (1971), no. 35-61. MR0279830 (43:5551)

8. J. Dydak and J. Segal, Shape theory. An introduction, Lecture Notes in Mathematics, 688, Springer, 1978. MR520227 (80h:54020)

9. R. W. Easton, Geometric methods for discrete dynamical systems, Oxford Engineering Science Series, 50, Oxford University Press, 1998. MR1616726 (2000e:37002)

10. A. Giraldo, M. A. Morón, F. R. Ruiz del Portal, and J. M. R. Sanjurjo, Shape of global attractors in topological spaces, Nonlinear Anal. 60 (2005), no. 5, 837-847. MR2113160 (2006k:37024)

11. A. Giraldo and J. M. R. Sanjurjo, On the global structure of invariant regions of flows with asymptotically stable attractors, Math. Z. 232 (1999), no. 4, 739-746. MR.1727551 (2000i:54063)

12. B. Günther and J. Segal, Every attractor of a flow on a manifold has the shape of a finite polyhedron, Proc. Amer. Math. Soc. 119 (1993), no. 1, 321-329. MR.1170545 (93k:54044)

13. S. Hu, Theory of retracts, Wayne State University Press, 1965. MR0181977 (31:6202)

14. A. Kadlof, On the shape of pointed compact connected subsets of $E^{3}$, Fund. Math. 115 (1983), no. 3. MR.708174 (85b:54053)

15. S. Mardešić and J. Segal, Shape theory. The inverse system approach, North-Holland Mathematical Library, 26, North-Holland Publishing Co., 1982. MR676973 (84b:55020)

16. J. W. Milnor, Topology from the differentiable viewpoint, The University Press of Virginia, 1965. MR0226651 (37:2239)

17. H. E. Nusse and J. A. Yorke, Characterizing the basins with the most entangled boundaries, Ergodic Theory Dynam. Systems 23 (2003), no. 3, 895-906. MR1992669 (2004g:37027)

18. D. Salamon, Connected simple systems and the Conley index of isolated invariant sets, Trans. Amer. Math. Soc. 291 (1985), no. 1, 1-41. MR797044 (87e:58182)

19. J. M. R. Sanjurjo, Multihomotopy, Čech spaces of loops and shape groups, Proc. London Math. Soc. (3) 69 (1994), no. 2, 330-344. MR1281968 (95h:55011)

20. _ On the structure of uniform attractors, J. Math. Anal. Appl. 192 (1995), no. 2, 519-528. MR1332224 (96c:58109)

21. E. H. Spanier, Algebraic topology, McGraw Hill Book Co., 1966. MR0210112 (35:1007)

22. J. E. West, Mapping Hilbert cube manifolds to ANR's: a solution of a conjecture of Borsuk, Ann. Math. (2) 106 (1977), 1-18. MR0451247(56:9534) 
Facultad de Matemáticas, Universidad Complutense de Madrid, 28040 Madrid, Spain

E-mail address: jajsanch@mat.ucm.es

Facultad de Matemáticas, Universidad Complutense de Madrid, 28040 Madrid, Spain

E-mail address: jose_sanjurjo@mat.ucm.es 